\title{
eJRIEPS
}

Ejournal de la recherche sur l'intervention en éducation physique et sport

46 | 2020

Varia

Critères d'identification d'entraîneurs experts. Proposition dans le contexte du haut niveau et de la préparation des Jeux olympiques

Identify expert high-level trainers to capitalize experience's knowledge.

Validation of ten criteria

Stéphane Fukazawa-Couckuyt et Jean-François Robin

C OpenEdition

Journals

Édition électronique

URL : http://journals.openedition.org/ejrieps/5026

DOI : 10.4000/ejrieps.5026

ISSN : 2105-0821

Éditeur

ELLIADD

Référence électronique

Stéphane Fukazawa-Couckuyt et Jean-François Robin, «Critères d'identification d'entraîneurs experts. Proposition dans le contexte du haut niveau et de la préparation des Jeux olympiques », eJRIEPS [En ligne], 46 | 2020, mis en ligne le 01 avril 2020, consulté le 21 novembre 2020. URL : http:// journals.openedition.org/ejrieps/5026; DOI : https://doi.org/10.4000/ejrieps.5026

Ce document a été généré automatiquement le 21 novembre 2020.

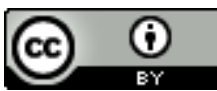

La revue eJRIEPS est mise à disposition selon les termes de la Creative Commons Attribution 4.0 International License. 


\section{Critères d'identification} d'entraîneurs experts. Proposition dans le contexte du haut niveau et de la préparation des Jeux olympiques

Identify expert high-level trainers to capitalize experience's knowledge. Validation of ten criteria

Stéphane Fukazawa-Couckuyt et Jean-François Robin

\section{Introduction}

1 Des résultats récents (Burlot, Delalandre, Joncheray, Demeslay, \& Julla-Marcy, 2019), congruents avec une étude antérieure (Lemieux \& Mignon, 2006), indiquent que le turn-over est très important pour les entraîneurs de haut niveau Français. Pour ceux présents aux Jeux olympiques de Rio, $50 \%$ sont en poste depuis $2012-2016$ et $25 \%$ depuis 2008-2012. Un quart seulement est en poste depuis au moins huit ans. Par ailleurs, l'entraîneur s'appuie principalement sur son expérience et celle de pairs pour prendre des décisions et agir (Burlot \& al., 2019 ; Cushion, Armour, \& Jones, 2003; Erickson, Côté, \& Fraser-Thomas, 2007 ; Fleurance \& Cotteaux, 1999; Gould, Giannini, Krane, \& Hodge, 1990 ; Lemieux \& Mignon, 2006 ; Loquet, 2011 ; Pérez, 2009 ; Reade, Rodgers, \& Hall, 2008 ; Saiz, Calvo, \& Ibanez Godoy, 2009). Le renouvellement élevé des entraîneurs couplé à l'importance de l'expérience amène à étudier et à mettre en place des projets de capitalisation des savoirs (Zacklad \& Grundstein, 2001) issus de l'expérience de ces entraîneurs.

2 En fonction du public visé par la diffusion des savoirs capitalisés (e.g. managers sportifs, étudiants, entraîneurs de haut niveau ${ }^{1}$, entraîneurs en contexte récréatif, etc.), 
le choix du profil des entraîneurs dont les savoirs vont être capitalisés diffère. Ici, la demande sociale a été de capitaliser des savoirs issus de l'expérience d'entraîneurs experts entraînant des sportifs de haut niveau, inscrits sur les listes ministérielles et préparant les Jeux olympiques. Comment identifier de tels entraîneurs? Dans cet article, des critères pour identifier des entraîneurs de haut niveau experts préparant les Jeux olympiques sont proposés, préalablement à l'étude sur la capitalisation des savoirs. La discussion autour de ces critères ouvre la réflexion sur l'identification d'autres profils.

3 À partir de la littérature générale sur l'expertise puis spécifique à l'entraîneur, une première version des critères d'identification d'entraîneurs de haut niveau experts a été proposée. Ces critères - théoriques - ont été vérifiés auprès de dix entraîneurs de haut niveau œuvrant dans une discipline olympique. S'en suit une discussion sur la pertinence de ces critères et une seconde version.

\section{Ancrage théorique}

Cette recherche se déroulant dans le contexte institutionnel et social de l'INSEP, l'Institut National des Sports et de l'Éducation Physique, le problème est de repérer des entraîneurs de haut niveau, qui soient garants d'une expertise et sources d'inspiration et de savoirs pour leurs pairs. Pour critérier cette identification de manière rationnelle et dans une perspective de reproductibilité scientifique et professionnelle, la littérature sur l'expertise est mobilisée.

\subsection{Caractériser l'expertise}

$5 \quad$ Ericsson est un des auteurs qui a défini ce concept en travaillant avec différents publics tels que des musiciens, des joueurs d'échecs ou des sportifs. Alors qu'avant l'expertise était considérée comme de l'ordre du don, du divin ou du surnaturel, les travaux de ce chercheur permettent de parler en termes d'habiletés acquises et de capacités innées. Ericsson et Charness (1994, p. 725) disent à cet effet que « recent research in different domains of expertise has shown that expert performance is predominantly mediated by acquired complex skills".

6 Cet apprentissage se fait non pas de façon automatique en agissant et en accumulant du vécu, mais «through structured learning and effortful adaptation » (idib). L'expérience est alors un préalable à l'atteinte d'une expertise (Ericsson \& Charness, 1994 ; Ericsson, Krampe, \& Tesch-Romer, 1993). Touboul (2011), dans sa thèse de doctorat en Sciences de l'Éducation portant sur les pratiques d'enseignement de la Savate Boxe Française en Éducation Physique et Sportive, fait état de la difficulté de différencier expérience et expertise, liées, imbriquées et complémentaires, dans ces pratiques. Il souligne toutefois que l'expérience seule ne suffit pas à développer l'expertise. Celle-ci se distingue de l'expérience dans le caractère «extrême »: les adaptations sont exceptionnelles, dans le cadre d'un effort de vie («life-long effort », Ericsson \& Charness, 1994, p. 744). Les adaptations se font en fonction des demandes et des besoins dans des domaines restreints et définis.

7 Du fait d'une extrême adaptation, l'expert réalise des performances au-dessus de celles de la moyenne des individus, voire exceptionnelles (Ericsson \& Charness, 1994; Macquet \& Fleurance, 2006), dans un domaine particulier. 
Comme le souligne Touboul (2011), évaluer l'expertise est difficile et se fait plutôt par inférence. Quels critères propose la littérature pour identifier des entraîneurs experts?

\subsection{Identifier l'entraîneur expérimenté et expert dans un contexte de haut niveau}

Côté, Salmela, Trudel, Baria, et Russell (1995) retiennent plusieurs critères pour identifier des entraîneurs de haut niveau experts : (a) être entraîneur depuis dix ans au minimum, (b) avoir entraîné au moins deux sportifs de niveau national et un de niveau international, seniors, (c) être reconnu par la fédération nationale comme un des meilleurs dans sa discipline pour entraîner des élites. Ces auteurs invitent à étudier des entraîneurs qui interviennent auprès de sportifs qui sont dans la dernière phase de développement du talent selon le modèle de Bloom (Bloom \& Sosniak, 1985), autrement dit, qui ont au moins 15 ans de pratique sportive et qui sont dans un groupe dont le quotidien tourne, en grande partie, autour de la performance sportive. Enfin, ils regardent également le niveau atteint par l'entraîneur dans son passé de sportif (régional, national ou international). Ces éléments sont repris par des études plus récentes. Afin d'identifier des entraîneurs de haut niveau experts, Lemyre, Trudel, et Durand-Bush (2007) indiquent trois critères génériques: (a) le nombre de victoires, (b) l'engagement à un niveau national et international et (c) dix ans ou 10000 heures comme entraîneur, sur la base des travaux fondateurs sur l'expert (Chase \& Simon, 1973 ; Ericsson \& al., 1993), confirmé dans le domaine du sport (Bloom \& Sosniak, 1985). Werthner et Trudel (2006) ont retenu des entraîneurs qui (a) ont coaché à un niveau national ou international de compétition pendant au moins dix ans, (b) ont été entraîneurs sur au moins une édition des Jeux olympiques et (c) sont présentement (au moment de l'étude) entraîneurs d'athlètes avec au moins un résultat au top 10 mondial dans les deux années passées. Pour parler d'expertise et d'expert, d'après Saury (2004), il faut être reconnu dans la durée et avoir entraîné dans des contextes différents. La reconnaissance sociale est un critère (Côté \& al., 1995 ; Rolland, 2011 ; Salmela, 1996), avec invitation de l'entraîneur à des conférences, formations, parrainages, etc.

\subsection{Synthèse}

Un expert est un individu qui réalise un effort de progression inscrit dans la durée, dans un domaine précis, basé sur l'expérience. Au fil du temps, ses performances deviennent supérieures à la moyenne. Cette expertise lui vaut le qualificatif d'expert, qualificatif attribué sur la base d'un jugement social. À ce titre, l'entraîneur-expert peut être défini comme un entraîneur qui contribue significativement à l'obtention d'une performance athlétique supérieure à celle des concurrents (pour le sportif). En reprenant les travaux sus-cités, quatre catégories de critères émergent pour identifier un entraîneur de haut niveau expert :

- le niveau de l'entraîneur comme sportif (Côté \& al., 1995) ;

- le vécu de l'entraîneur. Les auteurs parlent de dix années minimum comme entraîneur ou de 10000 heures d'entraînement pour être expert (Chase \& Simon, 1973 ; Ericsson \& al., 1993 ; Ericsson, Charness, Feltovich, \& Hoffman, 2006 ; Lemyre \& al., 2007). Il est aussi question du nombre de victoires des sportifs entraînés, ces victoires devant s'inscrire dans la durée et 
dans des contextes différents (Saury, 2004). Des auteurs évoquent également la participation comme entraîneur à au moins une édition des Jeux olympiques (Werthner \& Trudel, 2006) ;

- le niveau des sportifs entraînés. L'entraîneur doit avoir accompagné au moins un sportif de niveau international (Côté \& al., 1995), voire dans le top 10 mondial (Werthner \& Trudel, 2006). Ses sportifs doivent se situer dans leur dernière phase de développement, c'est-à-dire dans le haut niveau, avec au moins quinze années d'entraînement à leur actif (Côté \& al., 1995) ;

- la reconnaissance sociale. L'entraîneur doit être reconnu par sa fédération comme un des meilleurs du pays, être reconnu par les sportifs et les pairs (Côté \& al., 1995 ; Rolland, 2011 ; Salmela, 1996).

\section{Identifier un entraîneur de haut niveau expert dans le contexte du haut niveau et de la préparation des Jeux olympiques : un premier modèle-guide}

11 À ce stade, deux types de critères sont identifiés. Il y a des critères que nous appellerons " critères contextuels ", liés au terrain de recherche (INSEP/grand INSEP, contexte de haut niveau senior, préparation olympique) et non à l'expertise des entraîneurs qui œuvrent dans ce cadre :

- critère contextuel 1: entrainer dans une discipline olympique. Ceci en cohérence avec le projet de l'INSEP, qui consiste à optimiser au mieux la performance pour obtenir un nombre conséquent de médailles lors des grands événements et notamment des Jeux olympiques ;

- critère contextuel 2: avoir des sportifs en dernière phase de développement, qui sont inscrits sur les listes de haut niveau ;

- critère contextuel 3 : entraîner au moins un sportif de niveau international.

Ces critères permettent de repérer des entraîneurs de haut niveau entraînant dans une discipline olympique. Dans le contexte de notre recherche portant sur ce type d'entraîneur, ces critères sont des prérequis qui ne permettent pas d'apprécier à eux seuls l'expertise.

D'autres critères le permettent, ce sont des critères que nous appellerons «critères d'expertise » :

- critère d'expertise 1 : avoir au moins un sportif dans le top 10 mondial ;

- critère d'expertise 2 : avoir une reconnaissance sociale dans le cadre d'emploi ;

- critère d'expertise 3 : avoir une reconnaissance sociale en étant invité à des conférences, formations, parrainages;

- critère d'expertise 4 : avoir dix années ou 10000 heures de vécu comme entraîneur dans un contexte d'entraînement d'élites (niveaux nationaux/internationaux);

- critère d'expertise 5 : avoir au moins une participation à une édition des Jeux olympiques comme entraîneur ;

- critère d'expertise 6 : avoir entrâné avec des statuts/dans des contextes différents.

14 Pour ce modèle, nous n'avons pas retenu de la littérature le niveau comme ancien sportif, car le lien avec l'efficacité comme entraîneur est discuté (Fleurance \& Cotteaux, 1999 ; Sarremejane, Sano, \& Lémonie, 2010 ; Schempp, McCullick, Grant, Foo, \& Wieser, 2010). Certains entraîneurs jugent même comme un atout le fait de ne pas avoir de passé d'ancien sportif de haut niveau (Fleurance \& Cotteaux, 1999). 
15 En accord avec Touboul (2011), il est considéré que c'est en croisant les critères qu'il est possible d'identifier avec une forte probabilité un entraîneur expert. Ce modèle se veut un guide. Sa pertinence est étudiée à travers l'étude empirique menée.

\section{Approche méthodologique}

\subsection{Le terrain}

Le terrain de recherche a regroupé des Pôles France de l'INSEP et du Grand INSEP préparant des sportifs en vue des Jeux olympiques.

\subsection{La méthode}

17 Une première liste d'entraîneurs de haut niveau répondant aux critères contextuels et semblant répondre aux critères d'expertise a été établie. Les entraîneurs ont été rencontrés pour présentation de la recherche. Sur les douze entraîneurs rencontrés, dix ont été volontaires suite à cette rencontre.

Ils ont été interviewés lors d'entretiens de type life-story (Jones, Armour, \& Potrac, 2003), ce qui représente dix entretiens pour le présent corpus. Suivant le design de l'étude de Werthner et Trudel (2009), chaque entretien a démarré par une question ouverte: "Peux-tu nous narrer ton profil, comment tu en es venu à être entraîneur de haut niveau en Pôle France?». Ensuite, des questions ont été posées en fonction du guide d'entretien composé de thèmes et de questions issus des travaux de Côté (2006), Côté \& al. (2013) et Werthner \& Trudel (2009) sur le parcours de l'entraîneur : (a) son parcours sportif, (b) sa formation et (c) sa carrière professionnelle. L'objectif fut de vérifier à travers le parcours que chaque entraîneur pouvait être qualifié d'expert au regard du modèle-guide préalablement formalisé.

Dans le cadre de la triangulation des données, un ou plusieurs " tiers » de l'entraîneur (sportifs, collègues) ont été interrogés et des données dites convoquées (car déjà préexistantes à la recherche, Van Der Maren, 1996) sont mobilisées (textes écrits par l'entraîneur, articles de presse sur lui, etc.).

Le corpus est repris dans le tableau ci-dessous.

Tableau I : participants et corpus de données

\begin{tabular}{|l|l|l|l|}
\hline $\begin{array}{l}\text { Source de données/ } \\
\text { Entraîneur (initiales) }\end{array}$ & $\begin{array}{l}\text { Interview type } \\
\text { « life-story " }\end{array}$ & $\begin{array}{l}\text { Entretien avec } \\
\text { tiers }\end{array}$ & $\begin{array}{l}\text { Textes sur l'entraîneur } \\
\text { (données convoquées) }\end{array}$ \\
\hline GO & $1 \mathrm{~h} 16-21$ pages & Entraîneur-adjoint & 3 \\
\hline JPG & $1 \mathrm{~h} 7-21$ pages & Collègue & 3 \\
\hline FD & $1 \mathrm{~h} 34-22$ pages & Collègue + Sportif & 1 \\
\hline GH & $1 \mathrm{~h} 15-24$ pages & Aucun & 4 \\
\hline HO & $1 \mathrm{~h} 37-24$ pages & Collègue + Sportif & 3 \\
\hline
\end{tabular}




\begin{tabular}{|l|l|l|l|}
\hline PTP & $1 \mathrm{~h} 33-33$ pages & Sportif + Ex-sportif & 1 \\
\hline JF & 0 h $50-14$ pages & Collègue & 6 \\
\hline LMGC & $1 \mathrm{~h} 24-13$ pages & $\begin{array}{l}\text { Collègue } \\
\text { responsable de Pôle }\end{array}$ & 2 \\
\hline AC & $1 \mathrm{~h} 10-18$ pages & Sportif & 1 \\
\hline YM & $1 \mathrm{~h} 30-24$ pages & $\begin{array}{l}\text { Collègue + Ex- } \\
\text { sportif + } \\
\text { Ex-responsable }\end{array}$ & 2 \\
\hline
\end{tabular}

\subsection{Démarche des entretiens et précautions méthodologiques}

21 Avec une population absente du Pôle France entre 100 et 200 jours par an du fait du calendrier de stages et de compétition, chaque entretien a été convenu en avance tout en restant modifiable, selon les changements d'emploi du temps des entraîneurs.

Avant l'entretien, (a) l'usage des données, (b) les procédés d'enregistrement et de retranscription ainsi que $(\mathrm{c})$ « le thème et les objectifs » ont été rappelés (Van Der Maren, 1996, p. 313). Durant l'entretien, ce que la personne dit a été respecté ainsi que son rythme d'expression, sans critiquer, juger ou banaliser. Contrôlant nos paroles, pensées et sentiments, nous avons prêté attention à la relation dialogique en accord avec Faïta (1995), pour ne pas tomber dans l'écueil d'un professionnel qui cherche à s'exprimer dans les termes et catégories qui sont celles du chercheur.

\subsection{Le traitement des données}

Chaque entraîneur a reçu le verbatim de son interview pour relecture, pouvant demander des changements, retraits ou ajouts (Tracy, 2010). Après validation, deux codeurs ont identifié en double-aveugle le contenu à retenir. Tout en restant ouvert à d'autres données et catégories (approche inductive), deux codages de type déductif (Lodico, Spaulding, \& Voegtle, 2006) ont été réalisés :

- un premier codage reprenant les thèmes-questions de l'entretien: formation, parcours sportif, carrière professionnelle. Ce codage, en plus de faciliter le suivant, permet une vue d'ensemble du parcours de l'entraineur et a été utilisé dans le cadre de la capitalisation des savoirs qui suit la démarche d'identification des experts ;

- un second codage reprenant les six critères d'expertise et s'appuyant sur les données précédemment codées.

Un consensus de $85 \%$ minimum est attendu à l'issue du double-aveugle (Van Der Maren, 1996) et a été atteint. Puis, les différences restantes ont été discutées. Seuls les items faisant consensus ont été conservés. 


\section{Résultats}

Dans cet article, ne pouvant présenter les résultats dans leur exhaustivité, nous faisons le choix de trois axes de restitution. Tout d'abord, la présentation des résultats pour un des dix entraîneurs afin d'illustrer la démarche, à propos des catégories (a) «parcours sportif », (b) «formation» et (c) «parcours professionnel ». Puis, la présentation du profil de cet entraîneur au regard des six catégories que représentent les six critères. Enfin, une analyse transversale aux 10 entretiens, critère par critère. Notons que les entraîneurs sont identifiés par leurs initiales.

\subsection{Présentation d'un cas}

\subsubsection{Parcours de sportif}

Né en 1965, JPG a commencé le pentathlon par l'escrime (1972) et l'équitation (1976) ( $25^{\mathrm{e}}$ prise de parole de l'entraîneur JPG lors de l'interview : «JPG25 »), son père étant gendarme à la Garde Républicaine (JPG1). Puis il a fait du tir avec un gendarme entraîneur de l'US Métro. En seconde au lycée Charlemagne, ce monsieur lui parle du pentathlon et JPG se met à courir et nager (JPG1). Il intègre la section sports-études à Fontainebleau, pour deux ans, au sein de laquelle il devient champion de France junior avant de rentrer à l'INSEP en septembre 1983 (JPG1). Il y passe le bac en 1983 et est sélectionné pendant ses trois années de junior sur les trois championnats du monde (JPG2). Sénior à partir de 1987 (JPG2), il est champion de France en 1988 et remplaçant aux Jeux olympiques (JPG3). Jusqu'à la fin de sa carrière en 1997 (JPG6), il est régulièrement en équipe de France, participe à deux championnats du Monde, à deux championnats d'Europe et obtient une médaille par équipe aux championnats d'Europe (JPG4), en 1995 (JPG.SE2). Il est par ailleurs champion de France en 1995 et 1997 (JPG5). Il termine sa carrière $6^{\text {e }}$ d'une coupe du Monde en 1997 (JPG14).

Notons que sa vision actuelle d'entraîneur n'est pas sans lien avec son propre parcours dans le pentathlon. En effet, vers 1980 la Fédération a fait venir un entraîneur hongrois réputé, Lisman Laszlo, qui regroupe les meilleurs Français à l'INSEP (JPG28). JPG arrive en 1983 dans un collectif qui commence "à être huilé, avec un groupe, avec des installations à disposition et donc cet entraîneur-là qui avait déjà trouvé ses marques et qui est venu vraiment pour amener un vrai plus avec beaucoup d'exigence et de rigueur et qui s'est traduit quand même on peut le souligner par un titre de champion du Monde en 87 » (JPG28). Cet entraîneur a pris en compte le jeune JPG, alors en retrait et l'inspire (toujours). JPG était un pentathlète sans point fort et sans point faible (JPG31), avec à l'entraînement un niveau supérieur à l'escrime, mais une difficulté à le gérer en compétition en format "une touche » (JPG31/32/33). Ce manque de régularité en compétition sur l'escrime, prépondérante déjà à l'époque, lui fait rater des médailles tout au long de sa carrière internationale (JPG32). Son équitation est empreinte du monde militaire. Très académique, il manque d'habitude face aux obstacles, ce qui fait que les deux sports qu'il débute le plus tôt sont ceux où il est « peut-être le plus fragile » (JPG35). Concernant le tir, cette épreuve "vraiment technique voire même à dominante mentale» lui a toujours plu de par les difficultés qu'il y trouvait. Il a dû trouver des solutions en "gestion des émotions et gestion du stress " (JPG38), "par la démarche que cela demande, introspective de concentration. Et puis encore une fois c'est une épreuve où je me suis retrouvé en difficulté, donc c'est une épreuve où j'ai été obligé quand même d'aller chercher les solutions individuellement et 
j'y suis arrivé, donc finalement ça m'a beaucoup enrichi également » (JPG37). La natation lui a demandé beaucoup d'efforts, car il l'a commencé sur le tard (JPG39). Il trouve que finalement ces épreuves où il était en difficulté lui ont permis d'avoir une meilleure analyse et de construire des stratégies (JPG39).

\subsubsection{Formation} beaucoup aidé dans ma pratique de compétition » (JPG11), en 1996-1997 sur ses weekends (JPG12). Il s'abstient de faire de la sophrologie avec ses sportifs, en justifiant sa posture : pour lui, une relation particulière s'installe entre sophrologue et sportif, incompatible avec la relation entraîneur-entraîné (JPG83). Pour travailler avec un sophrologue, il faut que ce soit une personne distincte de l'entrâneur, comme cela a été fait sur 2004-2008 (JPG83). L'entraîneur recadre, « prend un rôle souvent pas facile quoi et de faire des séances de sophro ça peut faire un peu le mélange des genres et donc pour garder ce recul " (JPG84) il faut distinguer les rôles et missions (« on est là aussi pour taper sur les doigts, mettre des coups de pied au c** quand ça ne va pas, on n'est pas toujours le gentil », JPG85).

\subsubsection{Parcours de professionnel} trois fois par semaine, avec des adolescents (JPG13). Avant l'arrêt de sa carrière, il s'était positionné auprès de la fédération comme souhaitant devenir entraîneur de Pôle (JPG15). L'été 1997, suite à des changements fédéraux, un poste d'entraîneur adjoint à l'INSEP se libère (JPG15). Durant trois saisons il est l'adjoint de Christian Roudaut sur l'équipe masculine (JPG17/18), avec une responsabilité prononcée sur la natation et le tir. La transition - devenant entraîneur de sportifs qui étaient ses camarades et concurrents en équipe de France - s'est « très bien passée, je pense que j'ai su avoir la bonne posture [...] me mettre en retrait et eux ont eu l'intelligence de trouver leur intérêt également, je pense, parce qu'encore une fois sur la partie tir et la partie natation très rapidement c'était complémentaire par rapport à l'organisation du staff» (JPG19). Il estime que ces trois années ont été " essentielles », " peut-être les plus utiles dans sa construction d'entraîneur », car il a pu voir "vraiment le métier de près " sans être en «première ligne » (JPG19). Il croit «que souvent on lance des entraîneurs quand ils ne sont pas prêts"; lui a eu le temps de se préparer, et «l'entraineur est quand même une plaque tournante il est au milieu de tout, de voir toutes les problématiques de la fonction, et donc quand vraiment là on m'a proposé après les Jeux de 2000 de devenir le numéro 1 avec tout ce que cela peut comporter, et bien là j'étais prêt » (JPG22). Il devient donc en 2000 entraineur responsable des hommes, y compris de 2-3 juniors (JPG71). Comme d'autres entrâneurs qui n'ont pas connu les grands succès à l'international, il espère les obtenir en tant qu'entraîneur par le biais de ses sportifs (JPG68).

31 Il analyse sa longévité grâce aux évolutions, en plus de la variété de ce sport. Quand il est devenu entrâneur, une compétition se déroulait sur cinq jours, maintenant un seul. L'escrime est passée de $3 \mathrm{~min}$ à $1 \mathrm{~min}$, le tir à balles est passé à plomb puis laser, la

eJRIEPS, 46 | 2020 
course et le tir sont combinés (JPG79). Pour « un entraîneur c'est des révolutions et c'est des remises en question totales ", "c'est ce qui est le plus passionnant dans ce métier » (JPG80). Les athlètes changent, ce qui renforce la variété et la remise en question (JPG80). De plus, le fait d'être en relation constante avec des entraîneurs de course, tir, natation, escrime et équitation est important pour évoluer, "c'est passionnant c'est enrichissant et je pense que ça nous aide à garder aussi de la fraîcheur et de l'envie » (JPG86).

Il nous a communiqué les résultats de ses sportifs sur la période 2013-2016 (JPG.SE1).

Tableau II : les résultats de l'entraîneur

\begin{tabular}{|c|c|c|c|c|}
\hline $\begin{array}{ll}\text { Niveau } & \text { de } \\
\text { compétition } & \end{array}$ & $\begin{array}{l}\text { Coupe } \quad \mathrm{du} \\
\text { Monde }\end{array}$ & $\begin{array}{l}\text { Championnat } \\
\text { d'Europe }\end{array}$ & $\begin{array}{l}\text { Championnat du } \\
\text { Monde }\end{array}$ & $\begin{array}{l}\text { Jeux } \\
\text { olympiques }\end{array}$ \\
\hline Résultats & $\begin{array}{l}3 * \text { Or } \\
1 * \text { Argent } \\
7 * \text { Bronze }\end{array}$ & $\begin{array}{l}1^{*} \text { Or } \\
4 * \text { Argent }\end{array}$ & $\begin{array}{l}3 * \text { Or } \\
1 * \text { Argent } \\
1 * \text { Bronze }\end{array}$ & $4^{\mathrm{e}}$ et $20^{\mathrm{e}}$ places \\
\hline
\end{tabular}

Si dès 2008 certains des sportifs qu'il entraîne sont dans le top 10 mondial, avant les entretiens les titres ont été nombreux : «champion du Monde en 2013, on a été champion d'Europe également, en 2014 on a été vice-champion du Monde, vice-champion du Monde de relais, vice-champion d'Europe par équipe. Donc j'ai trois athlètes garçons qui sont dans le top 8 [...] 2 qui ont été déjà identifiés comme médaillables pour Rio » (JPG74).

\subsection{Codage en fonction des critères pour JPG}

Tableau III : adéquation critères de la recherche/profil de l'entraîneur

\begin{tabular}{|c|c|}
\hline $\begin{array}{l}\text { Critère d'expertise } 1 \text { : avoir au moins un } \\
\text { sportif dans le top } 10 \text { mondial ; }\end{array}$ & $\begin{array}{l}\text { Au moment des entretiens, il a trois sportifs dans } \\
\text { le top } 10 \text { mondial (JPG74; JPG.SE1). }\end{array}$ \\
\hline $\begin{array}{l}\text { Critère d'expertise 2: } \\
\text { reconnaissance sociale par le cadre } \\
\text { d'emploi ; }\end{array}$ & $\begin{array}{l}\text { En poste depuis quinze ans comme entraîneur- } \\
\text { chef (JPG22) après trois années comme adjoint } \\
\text { (JPG17), JPG a une grande stabilité } \\
\text { professionnelle. }\end{array}$ \\
\hline $\begin{array}{l}\text { Critère d'expertise } 3: \text { avoir une } \\
\text { reconnaissance sociale en étant invité à des } \\
\text { conférences, sur des formations, sur des } \\
\text { parrainages ; }\end{array}$ & $\begin{array}{l}\text { JPG a déjà été interviewé, invité pour une } \\
\text { conférence (JPG.SI1, JPG.SI2, JPG.SI4). }\end{array}$ \\
\hline $\begin{array}{l}\text { Critère d'expertise } 4 \text { : avoir dix années ou } \\
10000 \text { heures comme entraîneur dans un } \\
\text { contexte d'entraînement d'élites; }\end{array}$ & $\begin{array}{l}\text { JPG a quinze saisons de compétitions comme } \\
\text { entraîneur-chef et trois saisons comme } \\
\text { entraîneur adjoint à haut niveau (JPG15, 17, 71). } \\
\text { Cela représente plus de } 30000 \text { heures avec des } \\
\text { élites. }\end{array}$ \\
\hline
\end{tabular}




\begin{tabular}{|l|l|}
\hline $\begin{array}{l}\text { Critère d'expertise 5: avoir au moins une } \\
\text { participation à une édition des Jeux } \\
\text { olympiques comme entraîneur; }\end{array}$ & $\begin{array}{l}\text { Quatre participations de 2000 à 2012 comme } \\
\text { entraîneur (cinquième en 2016). }\end{array}$ \\
\hline $\begin{array}{l}\text { Critère d'expertise 6: avoir entraîné avec } \\
\text { des statuts/dans des contextes différents. }\end{array}$ & $\begin{array}{l}\text { JPG a entraîné en club (JPG12, 13) et en Pôle, du } \\
\text { loisir jusqu'à l'élite. Sur l'élite, il a été adjoint } \\
\text { (JPG15) puis responsable. }\end{array}$ \\
\hline
\end{tabular}

34 À la lecture de ce tableau reprenant les données issues de son verbatim au regard du codage avec les six critères, JPG est bien un « entraîneur de haut niveau expert ».

\subsection{Synthèse de l'interprétation des résultats pour le codage en fonction des critères}

Ici, il s'agit de regarder les critères retenus et les profils des entraîneurs de l'étude afin de voir en quoi les six critères sont validés ou non. Également, des éléments ressortent d'une analyse inductive, en marge des deux codages de type déductif. À partir de cet ensemble d'éléments, critère après critère, nous indiquons ce qui peut différer, soit entre les entraîneurs, soit entre un entraîneur et le critère en question.

\subsubsection{Critère d'expertise 1 « avoir au moins un sportif dans le top 10 mondial »}

Sur les dix entraîneurs interrogés, PTP n'a pas un sportif dans le top 10 mondial. S'il en forme, ces derniers vont au Pôle France olympique avant de performer dans le top 10 mondial. Par ailleurs, AC n'a pas non plus un sportif de ce niveau. Qu'est-ce qui fait que ces deux entraîneurs, sur ce critère, ne correspondent pas? Si AC est sur une génération montante avec laquelle il faut composer, pour PTP c'est son statut sur un Pôle France non olympique qui fait que d'une saison à l'autre les meilleurs éléments passent à l'INSEP. Il a formé plusieurs actuels top 10 Mondiaux. AC a quant à lui créé un Pôle France fédéral dont il assure la pérennité depuis plusieurs années. Aussi, si ces deux entraîneurs n'encadrent pas directement un sportif dans le top 10 mondial au moment de l'entretien, ils contribuent aux performances françaises dans leurs disciplines et ont une reconnaissance sociale croissante. Notons que peu après les entretiens PTP est devenu entraîneur au Pôle olympique. AC lui a accompagné les premiers champions du Monde français de sa discipline (2017).

Aussi, nous discuterons le critère « top 10 » au regard de la génération des athlètes mis à disposition des entraineurs de haut niveau tout au long de leurs carrières. De la même façon, le changement de statut et de place dans l'organisation est fréquent dans le sport de haut niveau et mérite d'être débattu.

\subsubsection{Critère d'expertise 2 « avoir une reconnaissance sociale par le cadre d'emploi »}

Sur ce point, il y a un consensus, leur statut d'entraîneur de haut niveau est reconnu depuis de nombreuses années. Suite aux Jeux olympiques de 2016, plusieurs de ces cadres ont eu une évolution de carrière, voire ont été recrutés comme entraîneurs à l'étranger ou dans une structure plus prestigieuse. 
4.3.3. Critère d'expertise 3 « avoir une reconnaissance sociale en étant invité à des conférences, sur des formations, sur des parrainages "

Si tous les entraîneurs ont une reconnaissance sociale à ce niveau, c'est hétérogène, entre des entraîneurs fortement sollicités, producteurs d'articles, d'ouvrages ou de DVD et des entraîneurs qui font uniquement des interventions ponctuelles en formation. Ce critère est donc peu discriminant dans cette étude.

\subsubsection{Critère d'expertise 4 " avoir dix années ou 10000 heures comme entraîneur} dans un contexte d'entraînement d'élites »

Sur ce point, tous remplissent un des deux critères, voire les deux. Seul PTP a au moment des entretiens neuf années et non dix et AC sept années, tout en ayant plus de 10000 heures d'intervention. Néanmoins, nous discuterons de ce seuil minimal, notamment au regard du continuum de l'expérience (Durand et al., 2013) et de la différence entre vécu et expertise.

\subsubsection{Critère d'expertise 5 « avoir au moins une participation à une édition des Jeux olympiques comme entraîneur »}

41 De nouveau, PTP et AC n'ont pas de participation comme entraîneur aux Jeux olympiques.

\subsubsection{Critère d'expertise 6 « avoir entraîné avec des statuts/dans des contextes différents »}

Sur ce point, tous ont eu des expériences dans des structures différentes (club; club labélisé Elite; Pôle Espoir; Association Sportive d'établissement scolaire; service municipal des sports ; équipe régionale), avec des niveaux différents, voire à l'étranger ou dans d'autres disciplines sportives.

\section{Discussion et proposition de critères revus}

43 Si les critères d'identification que nous avons retenus pour identifier des entraîneurs de haut niveau experts sont issus de la littérature, la démarche de cette étude est de les discuter.

\subsection{Critère d'expertise : avoir un sportif dans le « top 10 », une question de temporalité et de mission}

Pour des questions de génération et de place dans l'organigramme fédéral, AC et PTP n'avaient, au moment des entretiens, pas accès à un sportif dans le top 10 mondial. Ils œuvraient davantage sur la poursuite de la formation de sportifs en vue de leur permettre d'atteindre par la suite les meilleurs niveaux mondiaux, que sur un groupe déjà très performant. De par le cadre fédéral qui était le leur, ils ne répondaient pas, lors des entretiens, au critère d'avoir un sportif dans le top 10 mondial. Pourtant, l'année suivant son identification comme expert, AC a obtenu avec ses sportifs le 
premier titre français de champions du Monde dans sa discipline. PTP fut promu sur un autre Pôle France regroupant les Français performants dans le top 10 mondial dans cette discipline à catégories de poids. Cela interroge la validité du critère «avoir un sportif dans le top 10 mondial » (Werthner et Trudel, 2006) à travers deux points ; (a) la question de la temporalité de l'identification comme expert d'une part et (b) la mission de l'entraîneur.

Sur la temporalité de l'identification, un tel critère empêche l'attribution du qualificatif d'expert à un entraîneur comme AC qui obtient, l'année suivant les entretiens, un résultat lui permettant de répondre à ce critère. Pourtant, en regardant plus en détail son parcours, il est sur une dynamique de résultats croissants les années précédant son identification. Aussi, pour identifier un expert, il faut regarder ses résultats sportifs à l'instant $\mathrm{T}$, mais également leur dynamique sur les années qui précèdent. Également, un entrâneur qui accompagnerait un sportif jusqu'à la $11^{\mathrm{e}}$ place mondiale, dans un contexte où les meilleurs résultats français sont de l'ordre de la $30^{\mathrm{e}}$ place mondiale sur les deux dernières décennies, n'est pas identifié comme expert si l'on s'en réfère à ce critère. Pourtant il doit y avoir là une expertise. Cela invite à interroger la temporalité de nouveau, non celle des résultats de l'entraîneur, mais celle des résultats de la fédération. De la même façon, en prenant l'exemple d'un entraîneur qui accompagnerait un sportif en six mois de la $20^{\mathrm{e}}$ à la $5^{\mathrm{e}}$ place mondiale alors que ce dernier stagne depuis plusieurs saisons vers une $5^{\mathrm{e}}$ place, l'étude de la temporalité des résultats du sportif semble également à étudier.

Concernant la mission, un tel critère dans un contexte de sport de haut niveau écarte de l'identification comme "expert " les entraîneurs qui sont en charge des pôles et équipes de France espoirs et même des entraîneurs de séniors insuffisamment performants. Le manque de performance peut être multifactoriel et n'est pas systématiquement à attribuer à l'entraîneur tout comme la réussite (Saury, 2004). Cela invite donc à distinguer au regard des résultats deux sous-contextes dans le contexte élite identifié par Trudel et Gilbert (2006). Suite au récent rapport ministériel considérant différemment haut niveau et haute performance (Onesta \& al., 2018), nous proposons de considérer comme pouvant être expert tant l'entraîneur œuvrant dans le haut niveau que celui œuvrant pour que son sportif atteigne la haute performance « en montant sur le podium, voire ensuite en accédant à la plus haute marche [des podiums mondiaux]» (ibid., p. 10).

\subsection{Critère d'expertise : dix ans, 10000 heures et vécu}

Le concept des «dix ans/10 000 heures » est interrogé au regard de la littérature sur l'expertise. Ericsson et Charness (1994) disent que le développement de l'expertise se fait à travers un apprentissage structuré, des efforts délibérés, soutenus et réguliers visant à augmenter la capacité de performance selon les besoins, dans des domaines précis (Ericsson \& al., 1993). Aussi, le critère des dix années/10 000 heures, qui est un critère quantitatif de durée, ne permet pas, seul, d'inférer du développement de l'expertise chez l'entraîneur. Il peut être un repère, mais gagnerait à être précisé à l'aide d'indicateurs de développement de l'expertise par un apprentissage structuré, constant et orienté, tel que des pratiques réflexives de l'entraîneur (Bourgeois, 2013 ; Gilbert \& Trudel, 2006), la mobilisation d'outils favorisant la réflexivité (e.g. Cormery, 2013 ; Crinon \& Guigue, 2006 ; Wittorski, 2003). 


\subsection{Critère d'expertise : dix ans, 10000 heures et continuum de l'expérience}

48 «Dix ans/10 000 heures" de vécu professionnel dans le domaine considéré est un repère partagé par les scientifiques du champ (Chase \& Simon, 1973; Ericsson \& al., 2006, 1993 ; Lemyre \& al., 2007), ce pour quoi nous l'avons mobilisé pour cette étude. $\mathrm{Au}$-delà $\mathrm{du}$ point de discussion du paragraphe précédent sur les limites de l'appréciation quantitative du vécu, considérer uniquement le vécu professionnel se heurte au continuum de l'expérience (Durand \& al., 2013), qui est basé a minima sur le vécu (a) d'apprenant, (b) de sportif (c) et de professionnel.

49 Concernant le vécu d'apprenant en formation initiale, la littérature est critique sur son adéquation avec la réalité professionnelle (Bernardeau-Moreau \& Collinet, 2009; Cushion \& al., 2003 ; Fleurance \& Pérez, 2008 ; Pérez, 2009 ; Refuggi, Louis, Kossivi, \& Deridder, 2006). Néanmoins, sans conclure dans cet article, le fait de passer un diplôme d'État de 1200 heures en un an, un Master en cinq ans, de combiner plusieurs formations, de faire de la formation continue, n'est pas anodin dans la construction de l'expérience de l'entraîneur. Cela a probablement un effet sur les dix années et 10000 heures à prendre en compte.

50 La carrière passée de sportif est également considérée comme peu liée à l'efficacité professionnelle de l'entraîneur (Fleurance \& Cotteaux, 1999 ; Sarremejane \& al., 2010 ; Schempp \& al., 2010). Pour autant, on peut émettre l'hypothèse qu'un entraîneur qui a été sportif dans sa discipline trois années à raison de deux entraînements hebdomadaires n'a pas le même vécu qu'un entraîneur qui a pratiqué vingt années dont dix années internationales avec de l'entraînement biquotidien.

51 Enfin, nous avons pris en compte dans les dix ans et 10000 heures l'ensemble des expériences auprès de publics élites, sans forcément distinguer dans la dynamique des parcours les différents statuts (assistant, entraîneur, entraîneur principal, entraîneurchef), ni prendre en compte les expériences auprès de publics récréatif et perfectionnement du modèle de Trudel et Gilbert (2006). Aussi, il faudrait mieux étudier la construction de l'expertise à partir des parcours, fortement idiosyncratiques (Werthner \& Trudel, 2009).

52 Ces trois points autour du continuum de l'expérience amènent à préconiser une prudence et un questionnement qualitatif au-delà du critère quantitatif «dix ans/ 10000 heures ".

\subsection{Critère d'expertise non retenu : le niveau d'ancien sportif de l'entraîneur}

53 Au regard du débat scientifique sur le lien entre passé de sportif et compétence d'entraîneur, nous avons fait le choix de ne pas retenir le passé de sportif comme critère. Ce choix est conforté par les entretiens réalisés. Sur les dix entraîneurs experts identifiés :

- GO (GO.SE1 ; GO.SE2) et YM (YM3, 7), hautement reconnus dans leurs disciplines sportives, n'en sont pas issus. GO, entraîneur d'athlétisme, vient du basket-ball (niveau régional) et YM, entraîneur d'haltérophilie, a fait de la boxe anglaise, du bobsleigh (haut niveau) ;

eJRIEPS, 46 | 2020 
- LMGC, reconnu dans la communauté internationale d'après ses tiers (deux collègues) et ayant obtenu six médailles olympiques en 2016, a eu par le passé un niveau régional dans la discipline qu'il entraîne (MG.SE2). GH était joueur de nationale 2 dans sa discipline (GH3) ;

-JPG (JPG4 ; 6 ; 14), JF (JF.SE5 ; JF18), FD (FD.SE1 ; FD6), PTP (PTP28, 36, 37) et AC (AC24, 26, 27) sont dans leurs disciplines respectives d'anciens sportifs internationaux, jusqu'à un top 10 mondial, voire top 5 , pour certains ;

- Seul HO (HO7, 9 ; HO.SE1) est champion olympique et double champion du Monde. de recherches sur des professionnels intervenants à haut niveau (joueurs d'échecs, musiciens, sportifs d'élite, entraîneurs de haut niveau). Pour des recherches à venir, les six critères d'identification des entraîneurs experts doivent être reconsidérés si le contexte d'intervention n'est pas celui du haut niveau (les critères contextuels ayant changé, les critères d'expertise associés ne sont alors plus valables). Notamment, Lyle (2002) a préconisé de distinguer l'expertise selon le contexte d'intervention, ce qui a été repris par Trudel et Gilbert (2006) qui ont proposé de distinguer (a) le contexte récréatif (pratique sportive loisir), (b) le contexte du développement (pratique à un premier niveau de performance et de perfectionnement) et (c) le contexte élite (performance et compétitions internationales). Si déjà les critères d'expertise proposés sont à revoir pour différencier le contexte de la haute performance de celui du haut niveau, ils ne sont pas valides dans des contextes récréatifs ou de perfectionnement.

\subsection{Critère contextuel : des statuts d'entraîneur pluriels} sont pas head coach ont été identifiés comme experts. GH (GH26) est entraîneur-adjoint d'une équipe de France olympique dans un sport collectif ; LMGC (MG22), entraineur en sport de combat, n'est pas non plus head coach. Les huit autres entraineurs sont quant à eux entraîneurs-chefs. La littérature mobilisée autour de l'entraîneur de haut niveau expert ne précisait pas son statut au sein du staff. Pour autant, les adjoints peuvent ne pas être accrédités aux Jeux olympiques. Ce n'est pas le cas de GH et LMGC, mais ce fut celui de HO quand il était adjoint: il n'a pas pu être présent aux Jeux olympiques de 2012. Ainsi, le profil d'un entraîneur peut ne pas correspondre au critère d'expertise 5 , alors que l'entraîneur peut être expert en tant qu'adjoint. Ces résultats, qui sortent des catégories initiales, permettent de préciser un nouveau critère contextuel, le statut.

Sur le rôle et la place hiérarchique de l'entraîneur, l'International Sport Coaching Framework (ISCF) v1.2 (Conseil international pour l'excellence dans l'entraînement sportif \& al., 2013), rédigé par des scientifiques et des praticiens, propose de distinguer quatre statuts: (a) l'assistant, (b) l'entraîneur, (c) l'entraîneur avancé et (d) l'entraîneur-chef. Selon le statut, l'entraîneur n'a pas le même rôle et ne doit pas 
mobiliser les mêmes savoirs pour agir avec compétence. Nous faisons l'hypothèse que de ce fait, l'identification d'un entraîneur de haut niveau expert " assistant » diffère de celle d'un entraîneur de haut niveau expert « entraîneur-chef ».

\subsection{Proposition de néo-critères pour le modèle}

59 À l'issue de cette discussion, notre proposition initiale de six critères pour identifier des entraîneurs de haut niveau experts est reprise, ainsi que celle des critères contextuels. Les ajouts et nuances par rapport au modèle formalisé initialement dans cet article sont indiqués et mis en italique, y compris par rapport à des recherches futures et à des projets de capitalisation des savoirs issus de l'expérience d'entraîneurs.

Les critères contextuels portent notamment sur la discipline sportive, le contexte d'intervention (niveau des sportifs entraînés) et le statut de l'entraîneur;

- critère contextuel 1 : entraîner dans une discipline olympique. Si notre contexte et nos résultats n'apportent pas d'éléments à ce niveau, l'actualité autour des entrées et sorties de certaines disciplines dans le programme olympique (e.g. le surf), de disciplines de haut niveau, mais non olympique (e.g. Savate Boxe Française), de sports pratiqués localement (e.g. le hurling principalement pratiqué en Irlande) ainsi que l'existence de disciplines qui ne sont pas reconnues de haut niveau par les instances françaises (e.g. plongeurs du Red Bull Cliff Diving; sportifs professionnels; World's Strongest Man, etc.), amène d'autres possibilités de choix sur ce critère, pour d'autres études ou projets;

- critère contextuel 2: avoir des sportifs en dernière phase de développement, qui sont inscrits sur les listes de haut niveau. De nouveau, en marge de l'étude, ce critère peut être questionné pour des recherches ou des projets de capitalisation qui portent, par exemple, sur des athlètes en équipe de France cadet, en choisissant des sportifs masculins ou féminines, en ciblant des catégories d'âge, en portant sur des sportifs qui ne sont pas en dernière phase de développement;

- critère contextuel 3 : entraîner au moins un sportif de niveau international. Ce critère est directement impacté par le contexte d'intervention. Selon le positionnement dans un contexte haute performance, haut niveau, perfectionnement ou récréatif, ce critère est également à repenser, à recontextualiser;

- critère contextuel 4 : le statut de l'entraîneur, quel que soit le contexte d'intervention, est à préciser entre (a) l'assistant, (b) l'entraîneur, (c) l'entraîneur avancé et (d) l'entraîneur-chef. Plusieurs statuts peuvent être retenus, selon l'étude/le projet de capitalisation.

61 Ces critères permettent de repérer des entraîneurs de haut niveau entraînant dans une discipline olympique, ou de penser d'autres critères autour de la discipline, du contexte d'intervention et du statut de l'entraîneur, correspondant à d'autres contextes. Ces critères sont des prérequis contextuels qui amènent aux critères d'expertise dans cette recherche sur des entraîneurs experts. Suite à la discussion, les critères d'expertise sont repris pour le contexte qui est le nôtre, celui des entraîneurs de haut niveau et de haute performance experts entraînant dans une discipline olympique. Ainsi le modèle d'identification, qui se veut un guide, reprend les six critères d'expertise suivants :

- critère d'expertise 1: avoir au moins un sportif dans le top 10 mondial ou contribué à la formation d'un sportif qui a ensuite accédé au top 10 mondial, voire top 3 (haute performance). À noter que les entraîneurs peuvent être sur ces deux registres de la haute performance et de former à la haute performance, au sein de leur collectif d'entraînement. Tenir compte de la temporalité des résultats précédents pour mieux apprécier leur baisse, leur constance ou au contraire, le progrès amené dans les résultats par l'entraîneur ; 
- critère d'expertise 2: avoir une reconnaissance sociale dans le cadre d'emploi, comme entraîneur de haut niveau ;

- critère d'expertise 3 : avoir une reconnaissance sociale en étant invité à des conférences, formations, parrainages. À noter que ce critère est peu discriminant. Il est conservé pour mieux apprécier l'expertise ;

- critère d'expertise 4 : avoir dix années ou 10000 heures de vécu comme entraîneur dans un contexte d'entraînement d'élites (niveaux nationaux/internationaux). Au-delà de ce cadrage quantitatif impersonnel, tenir compte de l'expérience élaborée comme entraîneur de haut niveau et non du vécu; du parcours sportif, en formation, et de l'expérience élaborée dans d'autres contextes professionnels que celui d'entraîneur de haut niveau;

- critère d'expertise $5:$ avoir au moins une participation à une édition des Jeux olympiques comme entraîneur, si l'on cible ce type d'entraîneur. Ou avoir vécu au moins une olympiade intégralement, même sans avoir été aux Jeux olympiques. Critère repensé pour les entraîneursadjoints intervenants à haut niveau; les accréditations pour les Jeux olympiques sont toujours en nombre limité. Également pour les entraîneurs de haut niveau qui ne seraient pas sur une population des sportifs "qualifiés Jeux olympiques", pour diverses raisons (temporalité, génération, mission fédérale...);

- critère d'expertise 6 : avoir entraîné avec des statuts/dans des contextes différents. Ce critère rejoint la question du continuum et du développement de l'expérience.

\section{Conclusion} entraîneurs de haut niveau experts, afin de proposer un modèle nuancé, autour de deux catégories de critères: (a) les critères contextuels et les (b) critères d'expertise. À ce titre, elle sert le double enjeu (a) d'étayer des recherches qui porteraient sur un public d'entraîneurs de haut niveau experts et plus largement d'entraîneurs (b) de favoriser une identification plus claire et rationnelle des entraîneurs dont l'expérience sera capitalisée dans le cadre de projets de capitalisation des savoirs issus de l'expérience. Dans le champ du sport de haut niveau français, les besoins sont réels, car les dispositifs se structurent. Après le projet "Or olympique» (2012 à 2016) qui a consisté en la capitalisation de savoirs d'entraîneurs et d'athlètes qui ont obtenu au moins une fois l'Or aux Jeux olympiques dans leur carrière, l'INSEP structure différents dispositifs autour de la capitalisation des savoirs. Si deux d'entre eux sont à ce stade encore confidentiels, une lettre professionnelle a été récemment débutée sur un extranet privé destiné aux entraîneurs qui préparent les Jeux olympiques de 2020 et 2024. En dehors de l'INSEP, des initiatives telles que Les Cahiers des experts de la Fédération Française Handisport se développent.

Plusieurs axes de recherche sont identifiés à l'issue de cette étude, au-delà des différentes questions préalablement soulevées dans la discussion. Il s'agirait de :

1. mieux comprendre les parcours idiosyncratiques des entraîneurs ;

2. étudier l'interaction entre le choix d'un profil d'entrâneur pour capitaliser et le choix d'un public-cible auprès de qui les savoirs sont diffusés, pour mieux capitaliser et mieux réinvestir en formation les savoirs capitalisés. Par exemple, si l'objectif est de diffuser des savoirs auprès de futurs " entraîneurs-adjoints intervenants dans un contexte récréatif ", le choix d'un profil « entraîneur-chef intervenant dans un contexte haute performance » est discutable du point de vue de la cohérence des statuts et contextes d'intervention. Par ailleurs, pour un même statut et un même contexte d'intervention, envisager la 
capitalisation et la diffusion des savoirs/formation sous le seul angle des savoirs et des pratiques de l'expert, pour former des entraîneurs débutants par exemple, revient à considérer les pratiques des experts comme les meilleures pratiques (Lemyre \& al., 2007), selon un continuum de formation novice-expert (Trudel \& Gilbert, 2006) alors qu'il s'agit de pratiques efficaces pour des experts (Patton, 2001), dont la portée pour des débutants est discutable. Nous faisons l'hypothèse que l'on peut former avec pertinence des entraîneurs qui vont débuter dans le métier, à partir de savoirs issus de l'expérience d'entraîneurs expérimentés, mais non experts. Également, une personne peut ne pas être experte, mais être créative, innovante, ce qui est un enjeu dans la formation des entraîneurs (Krantz, 2008);

3. étudier l'existence, après les critères contextuels et les critères d'expertise, de « critères de capitalisation ». Par exemple, parmi les dix entraîneurs du panel de l'étude sur la capitalisation des savoirs, un des entraîneurs a eu des difficultés à verbaliser ses savoirs. Cela interroge sur ce que Barbier (2013) appelle les compétences de rhétorique de l'action ;

4. l'étiquette d'expert ne doit pas masquer les champs d'expertise, qui sont limités: un entraîneur qualifié d'expert est-il expert de tous les facteurs techniques, tactiques, contextuels, bioénergétiques, physiques, mentaux, nutritionnels de la performance? La littérature souligne le côté circonscrit et situé de l'expertise (Barbier, 2011 ; Nash \& Collins, 2006 ; Saury, 2004; Tochon, 1993) ; «l'expert » est en réalité expert sur un ou plusieurs points précis de l'entraînement. L'identification de ces points d'accroche de l'expertise est également un axe de recherche.

\section{BIBLIOGRAPHIE}

Barbier, J.-M. (2011). Vocabulaire d'analyse des activités. Paris : PUF.

Barbier, J.-M. (2013). Expérience, apprentissage, éducation. In L. Albarello, J.-M. Barbier, É. Bourgeois, \& M. Durand (Éd.), Expérience, activité, apprentissage (pp. 65-92). Paris : PUF.

Bernardeau-Moreau, D., \& Collinet, C. (2009). Les éducateurs sportifs en France depuis 1945 : questions sur la professionnalisation. Rennes : Presses universitaires de Rennes.

Bloom, B. S., \& Sosniak, L. A. (Éd.). (1985). Developing talent in young people (1 st ed). New York : Ballantine Books.

Bourgeois, E. (2013). Expérience et apprentissage. La contribution de John Dewey. In L. Albarello, J.-M. Barbier, É. Bourgeois, \& M. Durand (Éd.), Expérience, activité, apprentissage (pp. 13-38). Paris : PUF.

Burlot, F., Delalandre, M., Joncheray, H., Demeslay, J., Julla-Marcy, M., \& Menon, P. (2019). Enquête sur les conditions de travail des entraîneurs de haut niveau en France: des carrières au travail quotidien. Enquête statistique (p. 52). Paris : Ministère des Sports.

Chase, W. G., \& Simon, H. A. (1973). The mind's eye in chess. In W. G. Chase, Visual information processing (pp. 215-281). New York : Academic Press. 
Conseil international pour l'excellence dans l'entraînement sportif, Association des fédérations internationales des sports olympiques d'été, \& Leeds Beckett University. (2013). Cadre international pour la formation des entraîneurs. Illinois, Etats Unis: Human Kinetics.

Cormery, A.-C. (2013). Théories de l'expérience et pratique du journal. Questions vives recherches en éducation, 10(20), 95-105.

Côté, J. (2006). The development of coaching knowledge. International Journal of Sports Science and Coaching, 1(3), 217-222.

Côté, J., Erickson, K., \& Duffy, P. (2013). Developing the expert performance coach. In D. Farrow, J. Baker, \& C. MacMahon (Éd.), Developing elite sport performance : Lessons from theory and practice (pp. 17-28). New York : Routledge.

Côté, J., Salmela, J. H., Trudel, P., Baria, A., \& Russell, S. (1995). The coaching model : A grounded assessment of expert gymnastic coaches' knowledge. Journal of Sport et Exercise Psychology, 17(1), $1-17$.

Crinon, J., \& Guigue, M. (2006). Écriture et professionnalisation. Revue française de pédagogie, (156), 117-169.

Cushion, C. J., Armour, K. M., \& Jones, R. L. (2003). Coach Education and Continuing Professional Development: Experience and Learning to Coach. Quest, 55(3), 215-230.

Durand, M., Goudeaux, A., Horcik, Z., Salini, D., Danielian, J., \& Frobert, L. (2013). Expérience, mimesis et apprentissage. In L. Albarello, J.-M. Barbier, É. Bourgeois, \& M. Durand (Éd.), Expérience, activité, apprentissage (pp. 39-64). Paris : PUF.

Erickson, K., Côté, J., \& Fraser-Thomas, J. (2007). Sport experiences, milestones, and educational activities associated with high-performance coaches' development. The Sport Psychologist, 21, 302316.

Ericsson, K. A., \& Charness, N. (1994). Expert performance. Its structure and acquisition. American psychologist, 49(8), 725-747.

Ericsson, K. A., Charness, N., Feltovich, P. J., \& Hoffman, R. R. (Éd.). (2006). The Cambridge handbook of expertise and expert performance. Cambridge : Cambridge University Press.

Ericsson, K. A., Krampe, R. T., \& Tesch-Romer, C. (1993). The Role of Deliberate Practice in the Acquisition of Expert Performance. Psychological Review, 100(3), 363-406.

Faïta, D. (1995). Dialogue entre experts et opérateur : contribution à la connaissance de l'activité par l'analyse des pratiques langagières. Connexion, (65), 77-98.

Fleurance, P., \& Cotteaux, V. (1999). Construction de l'expertise chez les entraîneurs sportifs d'athlètes de haut-niveau français. Avante, 5(2), 54-68.

Fleurance, P., \& Pérez, S. (2008). Interrogations sur le métier d'entraîneur(e). Paris : INSEP.

Gilbert, W., \& Trudel, P. (2006). The coach as a reflective practitionner. In Robyn L Jones, The sports coach as educator : re-conceptualising sports coaching (pp. 113-129). London; New York: Routledge.

Gould, D., Giannini, J., Krane, V., \& Hodge, K. (1990). Educational needs of elite US national team, Pan American, and Olympic coaches. Journal of Teaching in Physical Education, 9(4), 332-344.

Jones, R. L., Armour, K. M., \& Potrac, P. (2003). Constructing Expert Knowledge: A Case Study of a Top-level Professional Soccer Coach. Sport, Education and Society, 8(2), 213-229. 
Krantz, N. (2008). Entraîner, un acte de création ? In P. Fleurance \& S. Pérez, Interrogations sur le métier d'entraîneur(e) (pp. 221-246). Paris : INSEP.

Lemieux, C., \& Mignon, P. (2006). Être entraîneur de haut niveau. Sociologie d'un groupe professionnel entre marché du travail fermé et marché du travail concurrentiel. (p. 171). Paris : Laboratoire de Sociologie du Sport, INSEP.

Lemyre, F., Trudel, P., \& Durand-Bush, N. (2007). How youth-sport coaches learn to coach. The Sport Psychologist, 21(2), 191-209.

Lodico, M. G., Spaulding, D. T., \& Voegtle, K. H. (2006). Methods in educational research: from theory to practice (1st ed). San Francisco, CA : Jossey-Bass.

Loquet, M. (2011). Knowledge-in-action between rules and experiences: lessons from high performance sport for physical education. Physical Education et Sport Pedagogy, 16(2), 145-162. https://doi.org/10.1080/17408981003712836

Lyle, J. (2002). Sports coaching concepts : a framework for coaches' behaviour. London : Routledge.

Macquet, A.-C., \& Fleurance, P. (2006). Des modèles théoriques pour étudier l'activité de l'expert en sport. Movement et Sport Sciences, (58), 9-41. https://doi.org/10.3917/sm.058.09

Nash, C., \& Collins, D. (2006). Tacit knowledge in expert coaching : Science or art ? Quest, 58(4), 465-477.

Onesta, C., Gilot, F., Henard, N., Le Fur, M.-A., Normand, B., \& Ourahmoune, S. (2018). Mission d'étude pour la haute performance sportive (p. 33). Ministère des Sports ; CNOSF ; CPSF.

Patton, M. (2001). Evaluation, Knowledge Management, Best Practices, and High Quality Lessons Learned. American Journal of Evaluation, 22(3), 329-336.

Pérez, S. (2009). Cognition et formation en sport de performance : de nouveaux cadres de pensée pour comprendre l'activité et la formation des cadres du sport de haut niveau? Intellectica, (52), 119-137.

Reade, I., Rodgers, W., \& Hall, N. (2008). Knowledge transfer: how do high performance coaches access the knowledge of sport scientists? International Journal of Sports Science and Coaching, 3(3), 319-334.

Refuggi, R., Louis, E., Kossivi, A., \& Deridder, M. (2006). Intervention et appropriation de « savoirs » sur les techniques sportives. Une innovation en formation universitaire des étudiants de licence STAPS. eJRIEPS, (9), 67-81.

Rolland, C. (2011). La connaissance des entraîneurs experts en gymnastique artistique : entre perception de formes et intelligibilité pratique. Blaise Pascal - Clermont-Ferrand II.

Saiz, S. J., Calvo, A. L., \& Ibanez Godoy, S. J. (2009). Development of expertise in Spanish elite basketball coaches. International Journal of Sports Science, 17, 19-32.

Salmela, J. H. (1996). Great job, Coach! Getting the edge from proven winners. Ottawa : Potentium.

Sarremejane, P., Sano, S., \& Lémonie, Y. (2010). De la culture du pratiquant à celle du praticien : la problématique de l'identification et de la théorisation du transfert de compétences. eJRIEPS, (20), 81-92.

Saury, J. (2004). Qu'est-ce qu'un entraîneur expert ? In J. Saury \& C. Sève, L'entraînement : les entraîneurs et leurs pratiques (pp. 35-50). Paris : Revue EP.S. 
Schempp, P. G., McCullick, B. A., Grant, M. A., Foo, C., \& Wieser, K. (2010). Professional Playing Experience Does Not Lead to Professional Coaching Success. Journal of Coaching Education, 3(3), 72-82.

Tochon, F.-V. (1993). L'enseignant expert. Paris : Nathan pédagogie.

Touboul, A. (2011). Les effets de l'expérience et de l'expertise dans les pratiques enseignantes en EPS : étude didactique clinique en Savate Boxe Française (Université Toulouse le Mirail-Toulouse II). Consulté à l'adresse https://tel.archives-ouvertes.fr/tel-00639543/

Tracy, S. J. (2010). Qualitative Quality: Eight « Big-Tent » Criteria for Excellent Qualitative Research. Qualitative Inquiry, 16(10), 837-851.

Trudel, P., \& Gilbert, W. (2006). Coaching and coach education. In D. Kirk, M. O' Sullivan, \& D. J. McDonald (Éd.), handbook of Physical Education (pp. 516-539). London : Sage.

Van Der Maren, J.-M. (1996). Méthodes de recherche pour l'éducation. Bruxelles ; Paris : De Boeck Université.

Vergnaud, G. (2010). Expérience et science s'opposent-elles ? Travail et apprentissage, (7), 33-47.

Werthner, P., \& Trudel, P. (2006). A new theoretical perspective for understanding how coaches learn to coach. Sport psychologist, 20(2), 198-212.

Werthner, P., \& Trudel, P. (2009). Investigating the idiosyncratic learning paths of elite Canadian coaches. International Journal of Sports Science and Coaching, 4(3), 433-449.

Wittorski, R. (2003). L'écriture sur la pratique comme outil de professionnalisation (la contribution de l'écriture sur la pratique professionnelle à la fabrication des savoirs et des compétences). In O. Douard (Éd.), Dire son métier : les écrits des animateurs (pp. 47-63). Paris : L'Harmattan.

Zacklad, M., \& Grundstein, M. (2001). Ingénierie et capitalisation des connaissances. Paris : Hermes science.

\section{ANNEXES}

\section{Annexe - Guide d'entretien}

Durée probable : Prévoir une à deux heures.

Rappel cadre d'entretien/enregistrement des données/droit de regard sur les données/ usage

Accroche :

«Si tu devais te présenter, quels sont les éléments que tu mettrais en avant?»

ou « A ton avis, qu'est ce qui t'a aidé à devenir un entraîneur ayant des réussites au plus haut niveau de performance?»

ou « Tu es entraîneur de haut niveau compétent et expérimenté ; à ton avis comment en es-tu arrivé là ?»

Thèmes-questions : 


\section{Thème $n^{\circ} 1$ : ma vie sportive}

- passé sportif (voir aussi le présent)

- entraîneurs ou gens qui l'ont marqué ? DTN, président, médecin, kiné, partenaire ou concurrent... Leader/mentor/personne qui influencent son parcours?

\section{Thème $n^{\circ} 2:$ ma vie professionnelle/expérience d'intervenant}

- ses contextes d'intervention et ses statuts

- ses expériences ou métiers qu'il juge essentiels

- les expériences qu'il juge avoir moins réussit et pourquoi

- les expériences qu'il juge avoir davantage réussit et pourquoi

- Leader/mentor/personnes qui influencent son parcours?

- Ses résultats

- Présentation de son contexte actuel d'intervention et de ses différentes missions : quel est son quotidien?

- Innovation, création, expérience, expertise

\section{Thème $n^{\circ} 3:$ mes études}

- formation informelle/formelle/non formelle - médiée, non médiée, réflexion interne/praticien réflexif/échanges avec autres entraîneurs. Cafétéria et autres lieux informels? Participe à des Formations Professionnelles Continues? Intervention sur des formations fédérales ? Médiathèques ? Internet...

- Leader/mentor/personnes qui influencent son parcours?

\section{NOTES}

1. http://www.sports.gouv.fr/pratiques-sportives/sport-performance/Sport-de-haut-niveau/ article/Le-sport-de-haut-niveau-c-est-quoi

\section{RÉSUMÉS}

Dans le cadre de démarches de capitalisation des savoirs issus de l'expérience auprès d'entraîneurs de haut niveau préparant les Jeux olympiques, la question des critères d'identification d'entraîneurs détenteurs d'une expertise est posée. À partir des travaux fondateurs sur l'expertise (Ericsson \& Charness, 1994) et de la littérature spécifique à l'expertise des entraîneurs de haut niveau (Côté, Salmela, Trudel, Baria, \& Russell, 1995; Werthner \& Trudel, 2006), un premier modèle d'identification d'entraîneurs de haut niveau experts est proposé, distinguant des critères "contextuels" et "d'expertise ». La pertinence de ces critères est validée par une recherche menée avec dix entraîneurs, avec une discussion et la proposition d'un second modèle guide, au regard (a) du statut de l'entraîneur et de ses évolutions de carrière et de résultats, (b) de la distinction de quatre contextes d'entraînement, récréatif, perfectionnement, haut niveau et haute performance (Onesta, Gilot, Henard, Le Fur, Normand \& Ourahmoune, 2018 ; Trudel \& Gilbert, 2006), (c) du continuum de l'expérience (Durand, Goudeaux, Horcik, Salini, Danielian \& Frobert, 2013) (d) et des « dix années/10 000 heures », critère quantitatif récurent dans la littérature sur l'expertise (Ericsson, Charness, Feltovich, \& Hoffman, 2006; Lemyre, Trudel 
\& Durand-Bush, 2007). En conclusion, des axes de recherches autour des parcours des entraîneurs et de la capitalisation de savoirs issus de l'expérience sont identifiés.

Within the context of capitalization of knowledge gained from experience with high-level coaches preparing for the Olympic Games, the question of the criteria for identifying coaches with expertise is raised. Based on foundational work on expertise (Ericsson \& Charness, 1994) and literature specific to the expertise of high-level coaches (Côté, Salmela, Trudel, Baria, \& Russell, 1995; Werthner \& Trudel, 2006), a first model for identifying high-level coaches is proposed, with "contextual" and "expert" criteria. The relevance of these criteria is validated by research conducted with ten coaches, with a discussion and the proposal of a second model guide, with regard to (a) the status of the coach and his career evolution and results (b) the recognition of four training context, recreation, development, high performance and high performance settings (Onesta, Gilot, Henard, Le Fur, Normand, \& Ourahmoune, 2018 ; Trudel \& Gilbert, 2006) and (c) the continuum of experience (Durand, Goudeaux, Horcik, Salini, Danielian, \& Frobert, 2013) (d) and "ten years/10,000 hours", a quantitative criteria in the literature on expertise (Ericsson, Charness, Feltovich, \& Hoffman, 2006; Lemyre, Trudel, \& Durand-Bush, 2007). In conclusion, areas of research around the careers of coaches and the capitalization of knowledge from experience are identified.

\section{INDEX}

Mots-clés : capitalisation des savoirs issus de l'expérience, entraîneur de haut niveau, modèle d'identification d'entraîneur, critères, expert, contexte d'intervention, continuum de l'expérience

Keywords : capitalization of knowledge gained from experience, high-level coach, coach identification model, criteria, expert, training context, continuum of experience

\section{AUTEURS}

\section{STÉPHANE FUKAZAWA-COUCKUYT}

Laboratoire LIRTES (Laboratoire Interdisciplinaire de Recherche sur la Transformation des pratiques Educatives et des pratiques Sociales, EA 7313), Université Paris-Est Créteil, France

\section{JEAN-FRANÇOIS ROBIN}

Laboratoire SEP (Sport, Expertise et Performance, EA 7370), Institut National du Sport, de l'Expertise et de la Performance, France 